Cómo citar este trabajo: Beato Bergua, S., Poblete Piedrabuena, M. Á., \& Cunill Artigas, R. (2019). Taxus baccata en la Sierra del Aramo (Macizo Central Asturiano). Boletín de la Asociación de Geógrafos Españoles, 81, 2772, 1-30. http://dx.doi.org/10.21138/bage.2772

\title{
Taxus baccata en la Sierra del Aramo (Macizo Central Asturiano)
}

Taxus Baccata in the Sierra del Aramo (Asturian Central Massif)

\section{Salvador Beato Bergua}

uo187213@uniovi.es

Departamento de Geografía

Universidad de Oviedo (España)

\section{Miguel Ángel Poblete Piedrabuena}

\author{
mpoblete@uniovi.es \\ Departamento de Geografía \\ Universidad de Oviedo (España)

\section{Raquel Cunill Artigas} \\ cunillraquel@gmail.com \\ Departamento de Geografía \\ Universitat Autònoma de Barcelona (España)
}

\section{Resumen}

Se analizan las formaciones vegetales con presencia de Taxus baccata situadas en la Sierra del Aramo, la composición florística de dichas comunidades, su distribución y estado. La investigación se ha fundamentado en el trabajo de campo y las herborizaciones fitosociológicas que han desvelado la relevancia de este taxón en el paisaje vegetal y la singularidad de algunos ejemplares y poblaciones. El estado de los tejos que forman parte del matorral petrano y la falta de brinzales en varias comunidades hace necesario plantear medidas para revertir su dinámica negativa. Asimismo, se ensaya el análisis pedoantracológico en dos ubicaciones por encima del límite 
superior del bosque, en zonas actualmente deforestadas. Los resultados demuestran la presencia de tejos en el Aramo desde la Edad del Bronce y ponen de manifiesto un cambio en el paisaje en consonancia cronológica con los hallazgos arqueológicos sobre la ocupación humana de las montañas del Macizo Asturiano.

Palabras clave: Taxus baccata; biogeografía; pedoantracología; Sierra del Aramo; Macizo Central Asturiano.

\begin{abstract}
The plant formations with presence of Taxus baccata located in the Sierra del Aramo are analyzed, in particular, the floristic composition of these communities, as well as their distribution and status. The research has been carried out through field work and phytosociological inventories. The relevance of this taxon in the vegetal landscape is revealed, as well as the singularity of some specimens and populations, while at the same time considering the necessary measures to reverse the negative dynamics of some communities in which it participates. On the other hand, the pedoanthracological analysis is tested from two pits made above the upper limit of the forest, in areas that are currently deforested. The results demonstrate the presence of yews in the Aramo since the Bronze Age and corroborate the archaeological findings about the human occupation of the mountains of the Asturian Massif.
\end{abstract}

Key words: Taxus baccata; biogeography; pedoanthracology; Sierra del Aramo; Asturian Central Massif.

\title{
1 Introducción
}

Taxus baccata es considerada una especie relicta en la península lbérica (Serra, 2009), que alcanzaba una mayor extensión en buena parte de Europa durante el último período glaciar (Thomas y Polwart, 2002). No obstante, debido a su capacidad para desarrollarse tanto sobre suelos profundos y ricos bien drenados como en ambientes rupícolas se ha adaptado y distribuido ampliamente por todo el continente (Ruiz de la Torre, 2006; Benham et. al, 2016). De hecho, el tejo forma parte del cortejo florístico de varias asociaciones vegetales tanto de la región mediterránea como eurosiberiana (Costa et al., 2001). Además, presenta algunas poblaciones en las que es la especie dominante y han sido caracterizadas como formaciones templadas secundarias y, en efecto, raras (Cortés et al., 2000). El taxón analizado está en la actualidad en regresión en diferentes lugares de Europa debido a la fragmentación de su hábitat y la escasa dispersión de las semillas, así como por los usos tradicionales y, sobre todo, por el ramoneo de ungulados (Tittensor, 1980; Svenning y Magård, 1999; García et. al, 2000; Dovciak, 2002; Thomas y Polwart, 2002; Mysterud y Østbye, 2004; Blanco et. al, 2008; Burgarella et. al, 2009; 
Sanz et. al, 2009; Díaz, 2010; Rodríguez et. al, 2011; Olmedo-Cobo y Gómez-Zotano, 2014; Campodrón et. al, 2016).

La cantidad de referencias a Taxus baccata en la bibliografía europea es bastante amplia (Abella, 2007, 2009a; Linares, 2013; Beato, 2018). Esta especie ha sido estudiada, entre otras, desde las perspectivas biológica, biogeográfica, botánica, ecológica y etnográfica, lo que implica que se ha abordado no sólo su comportamiento fitosociológico, evolución y dinámica, sino también su historia reciente y pasada y las interacciones con otros seres vivos y con los grupos humanos (García et. al, 2000; Lence et. al, 2011; Pérez-Díaz et. al, 2013; Uzquiano et. al, 2015). Si bien es cierto que los tejos y tejedas de la Cordillera Cantábrica han sido analizados en profundidad en algunas zonas, como en el extremo occidental (Rodríguez et. al, 2011), en la Sierra del Sueve (Álvarez et. al, 2006; Abella, 2009b) y en Asturias de forma general (Díaz, 2010), sin embargo, no se conoce con exactitud la distribución y el estado de este taxón en el Macizo Asturiano. La razón se debe, por un lado, a que no aparece representado en la cartografía publicada hasta la fecha y, por otro, a su consideración como especie secundaria en bosques y formaciones arbustivas.

Del mismo modo, se desconoce su evolución paleoecológica, toda vez que no se halla en ninguno de los análisis palinológicos realizados en territorio asturiano hasta la fecha (véase el completo recopilatorio y los datos propios de López-Merino, 2009). Incluso su relación pasada con los grupos humanos no ha sido analizada explícitamente en territorio asturiano. No obstante, Taxus baccata ha formado parte del legado sociocultural de muchos pueblos del viejo continente, incluso antes del Neolítico, y algunos especímenes han generado fascinación y veneración tradicionalmente (Cortés et. al, 2000; Díaz, 2010; Camprodon et. al, 2016). En este sentido, cabe señalar la importancia de un ejemplar en la Sierra del Aramo, el de Bermiego, declarado oficialmente Monumento Natural -declarado por Decreto 71/95, de 27 de abril- y cargado de un gran simbolismo, tal y como ocurre en otros tantos casos referidos a la especie. Se trata de un árbol con perímetro de 7,13 m y una edad estimada entre los 550 y los 800 años (es uno de los más viejos de Europa) distinguido en 2009 como "Árbol más longevo" por el Ministerio de Medio Ambiente, Rural y Marino (Díaz, 2010) y que ha sido candidato a árbol europeo en los años 2016, 2017 y 2018.

La presencia del tejo en la Sierra del Aramo ha sido citada académicamente (Navarro, 1976; Lence et. al, 2011; Beato, 2018) y en mayor medida en obras de divulgación. Además, cabe destacar los trabajos sobre la regeneración de Taxus baccata y las interacciones ecológicas en las que el Aramo ha servido de estación de muestreo y análisis (García et. al, 2000 y 2015; García y Obeso, 2003; García, 2006; Lavabre y García, 2015). Hasta la fecha, el primer acercamiento al conocimiento de la distribución del taxón en la sierra que incluye cartografía es el de Beato et al. (2018), que proponen la inclusión de los tejos del Aramo en el hábitat de interés comunitario 9580 para su protección. 
El objetivo de este trabajo ha sido aumentar el conocimiento biogeográfico sobre los tejos en el Macizo Asturiano a partir de dos líneas de investigación:

1. La del estado actual, mediante la caracterización fitosociológica de las formaciones vegetales con fuerte presencia de tejos (prestando especial atención a su estado de conservación) y la actualización de la cartografía sobre su distribución geográfica;

2. en el pasado, tratando de esclarecer la evolución holocena de Taxus baccata en Asturias en relación con los cambios antrópicos y naturales del paisaje.

\section{Material y métodos}

\section{1 Área de estudio}

La Sierra del Aramo es una montaña media calcárea del Macizo Central Asturiano, a $20 \mathrm{~km}$ al SW de Oviedo (Figura 1). Se extiende con una dirección dominante NNO-SSE a lo largo de unos 15 km de longitud entre el pueblo de Peñerudes (Morcín), al Norte, y el Alto de la Cobertoria, al Sur. Tiene 7 kilómetros de anchura aproximadamente y una altitud máxima de $1791 \mathrm{~m}$ en el Pico Gamoniteiru. Este espigón calcáreo separa las cuencas de los ríos Trubia al W y Caudal al E. Concretamente se trata de calizas namurienses rodeadas por materiales más deleznables del Devónico y, fundamentalmente, del Carbonífero westfaliense.

Su paisaje vegetal es el resultado de unos factores naturales de montaña oceánica (rigores climáticos, alta humedad, topografía abrupta, suelos esqueléticos, ubicación biogeográfica) pero, por supuesto, de una intensa antropización secular del territorio. Efectivamente, la explotación agroganadera campesina propició la extensión de superficies de pasto y castañedo (Beato, 2012), mientras que el fin de aquel sistema rural tradicional desde mediados del siglo XX trascendió en el abandono de prados y el crecimiento de los bosques y las áreas de matorral (Beato et. al, 2016a, 2016b). En este sentido, se están produciendo importantes transformaciones paisajísticas que no son abordadas convenientemente por la administración pública, poniendo en peligro un patrimonio natural y cultural, tan excelso como desconocido, como el de las comunidades vegetales con tejos de la Sierra del Aramo (Beato, 2018). 
Figura 1. Localización del área de estudio

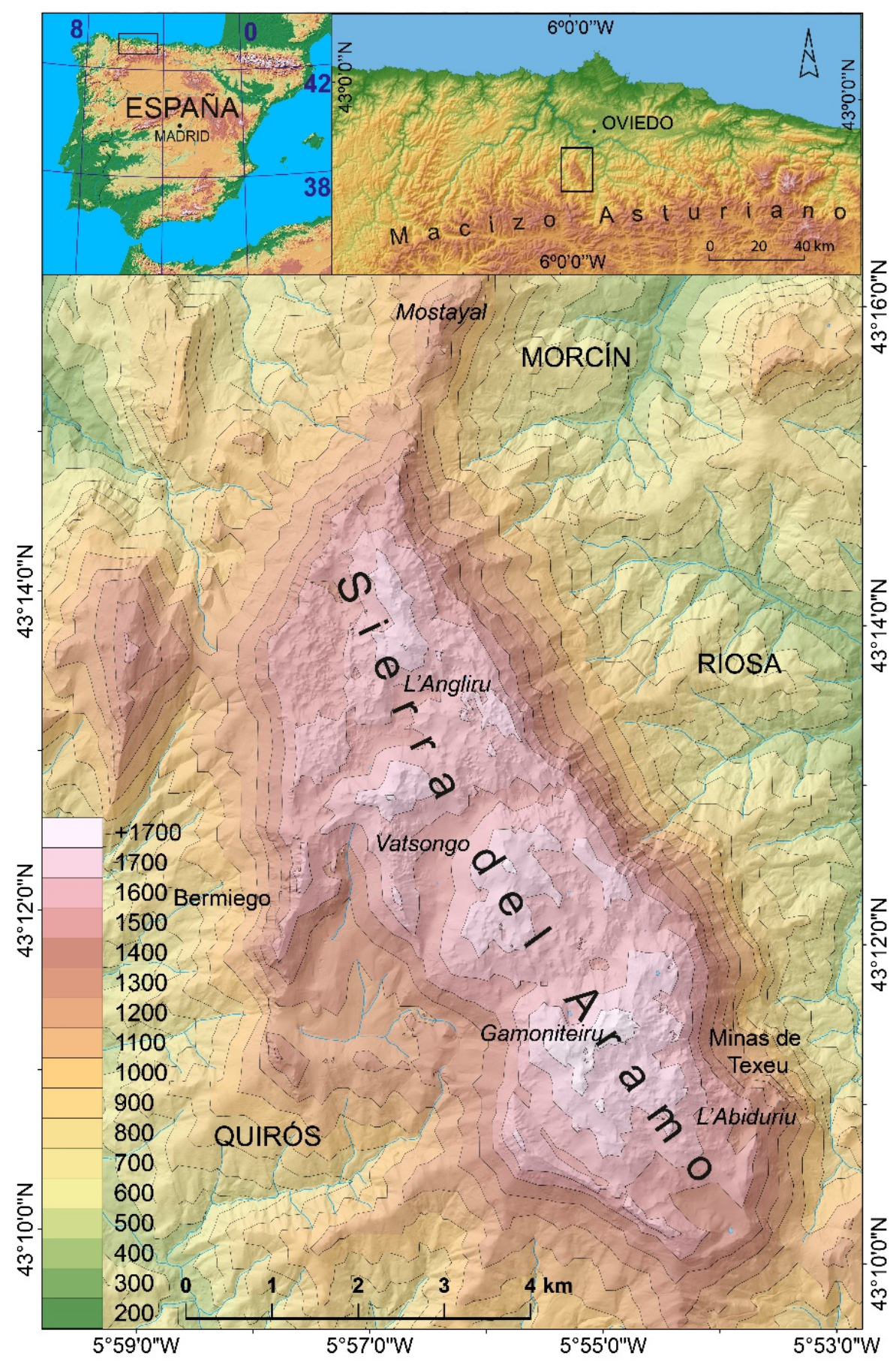

Fuente: elaboración propia a partir de las bases cartográficas digitales del Instituto Geográfico Nacional (Mapa Topográfico Nacional - MTN25 vectorial, Modelo Digital del Terreno - MDT05 y Modelo Digital del Terreno - MDT200)

\subsection{Caracterización geobotánica}

La metodología empleada se ha basado en el trabajo de campo con el fin de conocer la composición florística y estructura fisonómica de las formaciones vegetales con tejos, a tenor de 
criterios fitosociológicos de abundancia-dominancia de la especie (Braun-Blanquet, 1979; Fidalgo, 1997; Arozena y Molina, 2000); así como cartografiar dichas comunidades vegetales y realizar un mapa biogeográfico (Bertrand, 1966; Panareda, 1996). Durante el verano de 2017 se realizaron siete inventarios de vegetación en diferentes poblaciones de tejos presentes en la plataforma culminante del Aramo (seleccionadas previamente por su singularidad geobotánica y paisajística) para caracterizarlas fitosociológica, ecológica y dinámicamente. Se midió igualmente el perímetro de los ejemplares de Taxus baccata más longevos con el objetivo de catalogarlos e incluirlos en una base de datos propia y se observó in situ la presencia de señales de regeneración -nuevos brinzales, juveniles, semillas - o degradación -ramoneo, cortas, quemas, muertes- del taxón. Por otro lado, se consultaron los inventarios de Navarro (1974a y 1974b) y los de Beato (2018).

\subsection{Distribución espacial y cartografía}

Se ha elaborado un mapa a escala de representación 1:25.000 partiendo de la información cartográfica y las memorias del Mapa Forestal de España del Cuarto Inventario Forestal Nacional (2009), así como del Mapa Temático de Vegetación del Principado de Asturias (1994) y de los mapas y cliseries de vegetación de trabajos especializados sobre la zona de estudio (Navarro, 1974a y 1974b; Beato, 2018; Beato et al., 2017 y 2018). Posteriormente, se han corregido y precisado los límites espaciales de los diferentes elementos que constituyen el mosaico vegetal a partir de la fotointerpretación de los ortofotomapas del PNOA -años 2006, 2011 y 2015-, mediante el software informático Arcgis 10.1 y atendiendo a criterios de color, textura, localización y dinámica. Además, se ha efectuado la fotointerpretación de imágenes aéreas - Vuelo Americano 1956-1957, Vuelo Interministerial 1973-986, Vuelo Nacional 1980-1986 y Vuelo del PNOA 2014 - y de fotografías de paisaje, teniendo en cuenta la información aportada por miembros de instituciones de comunidades locales y lugareños; que han contribuido a valorar la dinámica reciente de las formaciones vegetales a tenor de los cambios socioeconómicos.

\subsection{Análisis pedoantracológico}

Por otra parte, para averiguar si el tejo ha estado presente desde antiguo en la Sierra del Aramo, se realizaron dos fosas pedoantracológicas a 1701 (ARAMO 1) y $1611 \mathrm{~m}$ (ARAMO 2) respectivamente, en dos depresiones kársticas colmatadas de arcillas y actualmente deforestadas. El objetivo era, además, obtener datos por encima de los $1600 \mathrm{~m}$ que marcan el límite superior del bosque actual y servir como sondeos preliminares en el marco de un proyecto más amplio geográficamente que analice el proceso geohistórico de generación de pastos. La primera fosa ARAMO 1- se excavó en la cara Sur del Gamoniteiru en una dolina colmatada por arcillas de descalcificación y pequeños depósitos de bloques movilizados desde los afloramientos rocosos circundantes. La vegetación actual es el pastizal subalpino moderadamente acidificado con algunas matas dispersas de matorral petrano, Daphne laureola básicamente. Se excavó hasta una 
profundidad de $160 \mathrm{~cm}$ para obtener un corte limpio de $140 \mathrm{~cm}$, pero no se consiguió llegar hasta el fondo del depósito. El perfil edáfico constaba de un horizonte A de $30 \mathrm{~cm}$ y una pequeña transición de $10 \mathrm{~cm}$ hacia la roca madre, esto es, las arcillas de descalcificación. Las características del suelo se correspondían con una formación de tipo Eutrudept según la clasificación de suelos de Asturias de Rodríguez Rastrero (2015).

La segunda fosa -ARAMO 2- se efectuó en la zona cimera al NE de El Angliru, en una depresión abierta con cierta inclinación hacia el $\mathrm{N}$, cubierta por arcillas y bloques, con pasto montano acidificado y un tanto nitrificado. Los afloramientos rocosos circundantes presentan matas de Genista hispanica subsp. occidentalis y vegetación casmófita con Ribes alpinum, Berberis vulgaris subsp. cantabrica y Rhamnus alpina. Se alcanzó la roca madre profundizando hasta los $100 \mathrm{~cm}$, obteniéndose un perfil limpio de $90 \mathrm{~cm}$. Su descripción es sencilla: un suelo Cryorthent típico con un horizonte A de 10-15 cm y una transición mínima a las arcillas de descalcificación con un mayor contenido rocoso hacia el fondo.

El método empleado ha sido el propuesto por Cunill (2010) y Cunill et. al (2013), que consiste básicamente en excavar una pequeña zanja hasta alcanzar la roca madre, la descripción de perfil edáfico y la extracción de muestras completas de suelo en todos los niveles (entre 5 y $10 \mathrm{~kg}$ por nivel). Se procede después a su secado y pesado, para ser tamizadas en húmedo con tamices homologados de $50 \mathrm{~cm}$ de diámetro y luces de malla de $5 \mathrm{~mm}, 2 \mathrm{~mm}$ y 0,8 mm.

La obtención de los fragmentos de carbón se realiza a través de la lupa binocular y la identificación de las especies mediante la interpretación de la anatomía de la madera a través del microscopio óptico -con luz reflectante episcópico- con lentes de magnificación de 100x, 200x y 500x- y con la ayuda de los atlas de anatomía de la madera (Schweingrüber, 1990a y 1990b; Vernet et. al, 2001), así como las colecciones de referencia del laboratorio GEODE de la Universidad de Toulouse II y del grupo i-Ge\&ser del Departamento de Geografía de la Universidad de Oviedo.

El perfil de la fosa ARAMO 1 se dividió en seis niveles de los que se extrajeron muestras con un peso total de 34,1 kg en seco. De ARAMO 2, por su parte, se extrajeron cinco niveles que, una vez secados para obtener el peso sin agua de la muestra (y que tengan mayor resistencia a los carbones), pesaron en conjunto $36,4 \mathrm{~kg}$. Los materiales acopiados fueron tamizados en húmedo y una vez secado lo obtenido en cada tamiz fue clasificado y guardado para el posterior aislamiento de los fragmentos de carbón. Tras identificar los carbones se pesaron de forma individual todos los fragmentos de carbón en una báscula de precisión, con la finalidad de calcular la antracomasa, esto es, la relación entre los carbones -en mg- y el material tamizado sin la fracción superior a 5 mm (en kg). Además, se dataron por carbono 14 -AMS radiocarbono- siete fragmentos de carbón de las dos fosas en el Poznań Radiocarbon Laboratory -Polonia-. La selección de las piezas se realizó en función de los taxones identificados y su posición en el perfil del suelo 
(profundidad), así como por el peso -el mínimo para poder ser datado es de 4 mg-. Las fechas de radiocarbono se han calibrado utilizando el programa OXCAL 4.3 (Bronk, 2017) y la curva de calibración IntCal13 (Reimer et al., 2013).

\section{Resultados}

\subsection{El tejo en las grandes formaciones vegetales del Aramo}

En la actualidad, Taxus baccata se halla en todas las vertientes de la Sierra del Aramo, en las diferentes formaciones vegetales que pueblan los diversos ambientes topoclimáticos de sus laderas (Figura 2). Efectivamente, se encuentra en comunidades que tradicionalmente se han asignado a varias asociaciones. Por un lado, en los castañedos naturalizados y carbayedas con abedul Quercus robur, Betula celtiberica, Castanea sativa- que se desarrollan sobre suelos lavados o ácidos en la transición colino-montana, en los que el tejo forma parte del sotobosque y juega un papel poco relevante. Por otro, en los bosques mixtos planocaducifolios montanos y calcícolas Fagus sylvatica, Betula celtiberica, Sorbus aria, Sorbus aucuparia, Salix caprea, Ilex aquifolium, Corylus avellana- en los que el tejo aparece en todos los estratos, tanto con forma arbustiva, como arborescente y arbórea, con una relevancia mayor.

Igualmente, este taxón participa de manera relativamente importante en las acebedas que prosperan colonizando los pastizales de las laderas, en formaciones de porte arbustivo y arborescente dominadas por llex aquifolium y Crataegus monogyna. No obstante, alcanza su mayor expresión en el sotobosque de los hayedos, especialmente los eutrofos - de la serie Carici sylvaticaeFagetum sylvaticae-. De hecho, en las áreas más elevadas y con suelos esqueléticos llega incluso a cuestionar el dominio del haya y conforma auténticos bosques mixtos en los que Fagus sylvatica, Taxus baccata y Sorbus aria alcanzan gran porte y densidad (Figura 3). En puridad, la plataforma cacuminal, en buena medida deforestada, presenta un buen número de comunidades vegetales dominadas por el tejo o, por lo menos, con un rol principal. Son, en general, formaciones arbustivas abiertas, incluso dispersas y rupícolas en algunos casos - matorral petrano- aunque destacan dos singulares excepciones: los bosques de L'Abiduriu y Vatsongo. 
Figura 2. Localización de las formaciones vegetales con tejos y las dos fosas pedoantracológicas

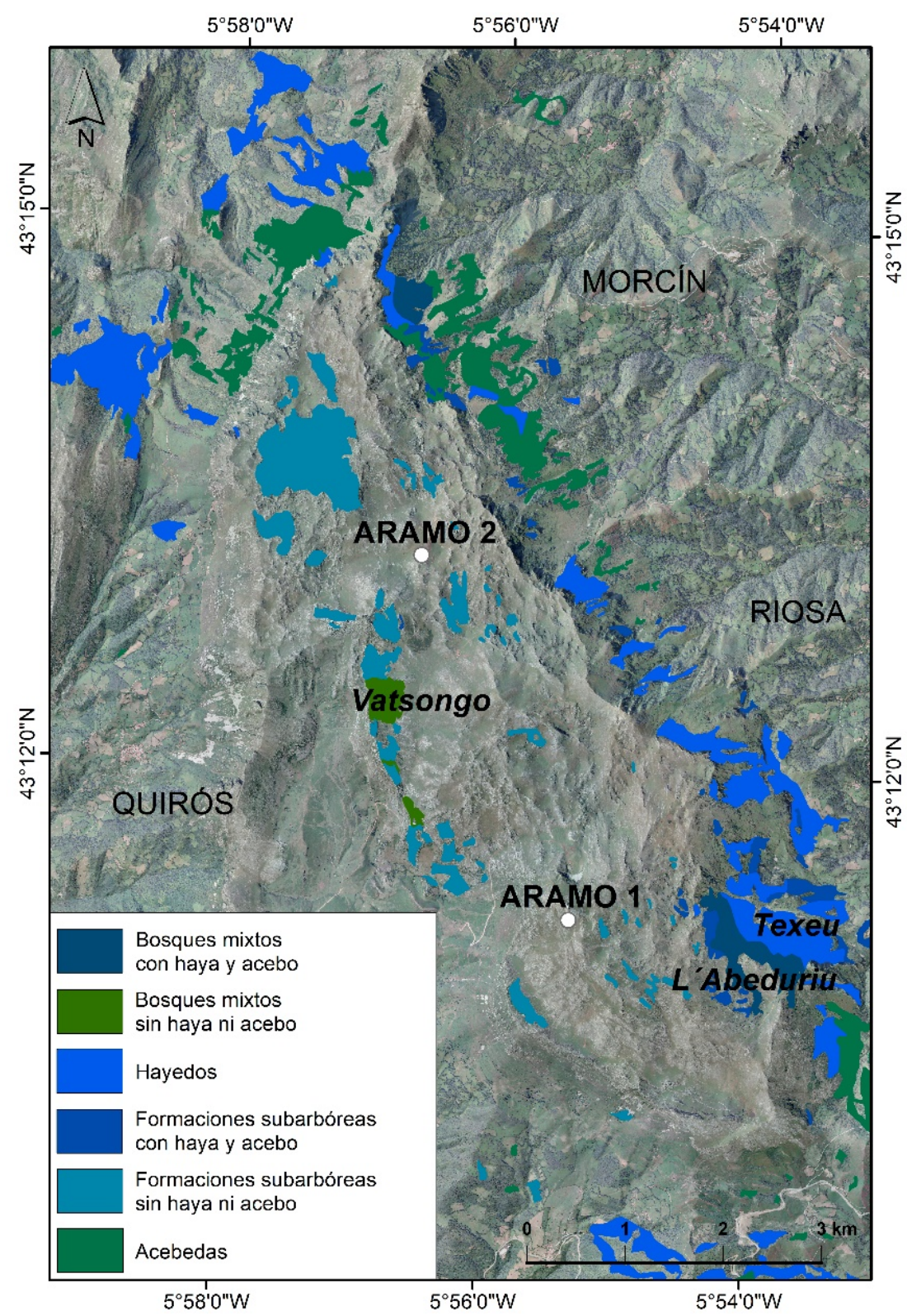

Fuente: elaboración propia a partir de Beato et. al (2018) y las bases topográficas digitales del Instituto Geográfico Nacional (Ortofotomapa PNOA 2015 y Modelo Digital del Terreno - MDT05)

Figura 3. Hayedo de Brañavieja con abundancia de tejos y mostajos

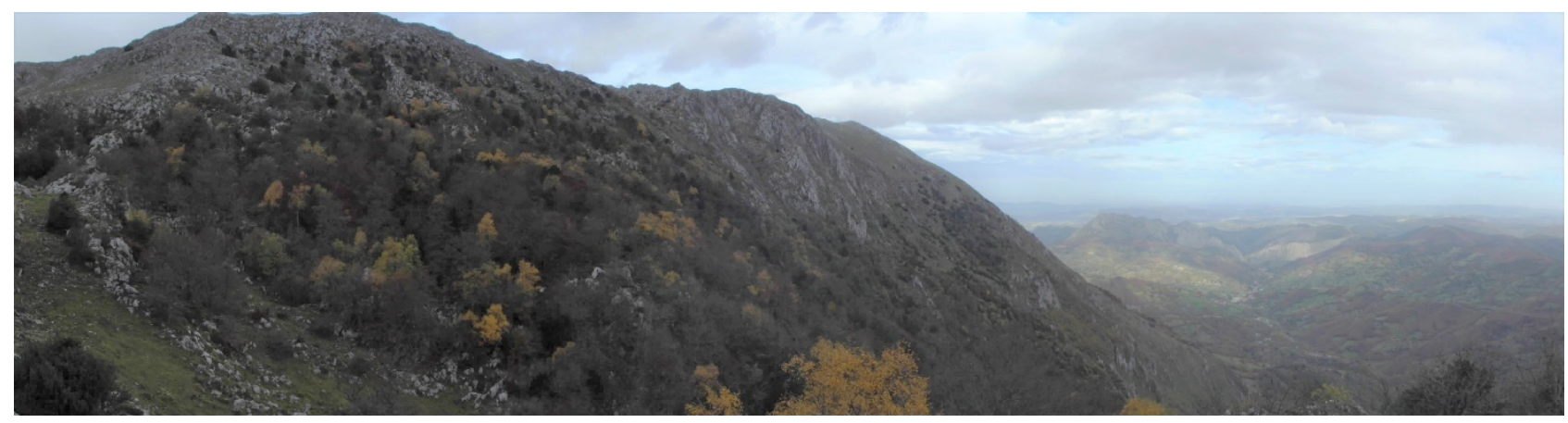

Fuente: elaboración propia 


\subsection{Análisis fitogeográfico de comunidades singulares con tejos}

Taxus baccata está prácticamente en todas las formaciones arbustivas y rupícolas, siendo difícil no encontrar algún ejemplar aislado en cualquier parte de la plataforma culminante. Teniendo en cuenta estos tejos de escaso porte que revisten las rocas de la zona cacuminal del Aramo, podemos señalar que nos hallamos ante una de las mayores representaciones de tejos de la península lbérica con una población muy por encima del millar de ejemplares, aunque con la cautela necesaria por las dificultades del censo y la escasez de datos para efectuar una comparación. En efecto, son múltiples las formaciones vegetales de porte herbáceo o subarbustivo dominadas por tejos ramoneados, convertidos en bonsáis macizos que tapizan la roca del orden de $1 \mathrm{~m}^{2}$ cada $10 \mathrm{~m}^{2}$ de parcela.

Además de los tejos, los roquedos de la plataforma culminante del Aramo están poblados por vegetación casmofítica, matorrales propios de los aulagares calcícolas y herbáceas procedentes de formaciones de pastizal o cespitosas. Tiene relativa importancia asimismo el brezal y las arbustedas subalpinas con Rhamnus alpina, Berberis vulgaris y Juniperus alpina. Por tanto, es difícil caracterizar esta comunidad de matorral petrano con tejos debido a los múltiples matices que encontramos en esta sierra. Por un lado, llama la atención la falta de llex aquifolium en buena parte de las arbustedas, salvo en L'Abeduriu y en otras pequeñas manchas dispersas. En estas últimas áreas, el tejo está acompañado por Rhamnus alpina y, en menor medida, por Juniperus alpina con brinzales juveniles de acebo y haya. La composición florística es claramente rupícola con abundancia de Saxifraga paniculata o Asplenium trichomanes, así como de otras plantas más ubicuas tales como Euphorbia flavicoma e Hippocrepis comosa. Por otro, la ausencia del acebo suele coincidir en otros lugares con la abundancia de Corylus avellana, especialmente en la mitad septentrional y en aquellas áreas donde la vegetación superior está aumentando en densidad y porte. Esto se debe a una menor presión ganadera merced a la prohibición de los fuegos y a una carga más especializada en el vacuno (las cabras son potencialmente mayores consumidoras de las matas y arbolillos que afloran en el roquedo). Por ejemplo, entre los picos Moncuevu - $1718 \mathrm{~m}-\mathrm{y}$ Vatsongo -1623 m- hay numerosas formaciones arbustivas eutrofas sobre los afloramientos del roquedo con dominio de Corylus avellana y gran abundancia de Taxus baccata (bien representado en un pobre estrato superior y en su forma tapizante), Rhamnus alpina y Sorbus aria (Figura 4). Desde el punto de vista fitosociológico, podría corresponderse, a tenor de los taxones mencionados y Berberis vulgaris y Rosa sp., con la asociación Pruno spinosae-Berberidetum cantabricae subas. taxetosum baccatae dados los taxones mencionados, así como Berberis vulgaris y Rosa sp. Sin embargo, no se han inventariado las características Prunus spinosa y Amelanchier ovalis y el dominio no corresponde a Rhamnus alpina sino a Corylus avellana. 


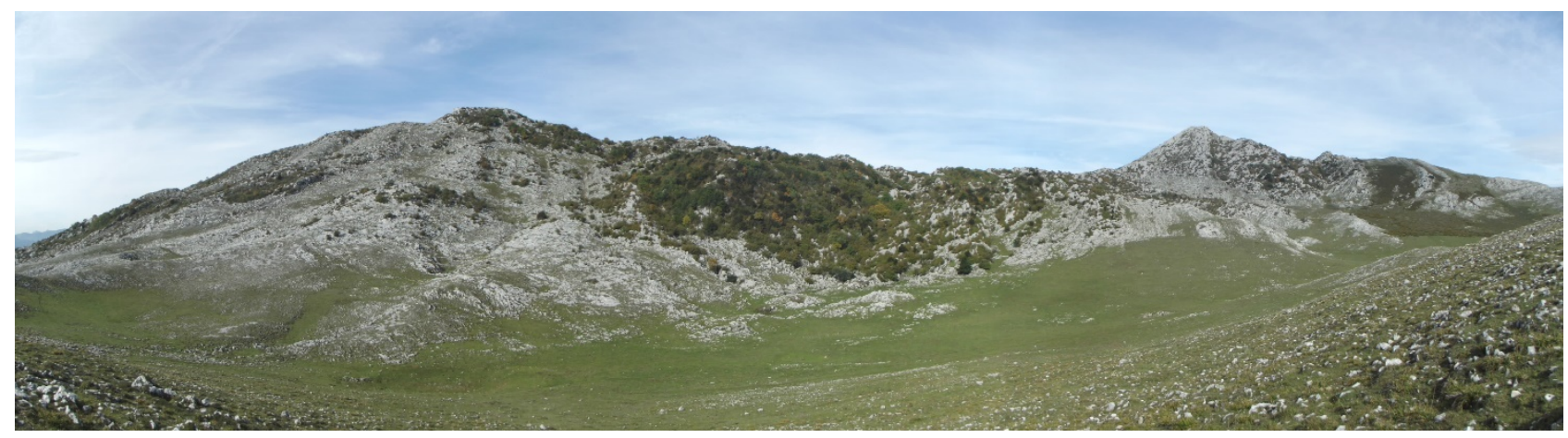

Fuente: elaboración propia

Este tipo de formación vegetal arbustiva y rupícola se dispone en forma de orla en torno al bosque mixto de Vatsongo. Se trata de un excelente ejemplo de comunidad arbórea rica en especies, de gran densidad y singularidad, aunque escasa extensión (8 ha). Se ha conservado sobre un roquedo calizo extremadamente karstificado y poblado de pozos nivales, lapiaces en agujas y dolinas de escarpadas paredes, en las que el tejo encuentra un refugio perfecto frente a los animales. Cabría pensar en su relación con el hayedo eutrofo por el sustrato litológico y la altitud, pero no se ha encontrado ni un solo ejemplar de haya ni de acebo en la zona.

Dentro de este bosque existen importantes doseles de tejo como especie dominante, mientras que en otros se hayan dispersos y priman Tilia platyphyllos, Betula celtiberica o Acer pseudoplatanus. En este caso, el estrato arbóreo es más variado aún, con ejemplares también de Sorbus mougeotii y algunos de Ulmus glabra. Tiene, por tanto, un carácter pluriespecífico y los estratos inferiores no ayudan a su clasificación fitosociológica, pues presentan vegetación propia de diferentes ambientes montanos -rupícolas, cosmopolitas, de pastizal y bosque eutrofo-. De este modo, por debajo de la cubierta arbórea se desarrollan pies o plantones juveniles de las planocaducifolias mencionadas, además de Corylus avellana, Sorbus aria, Sorbus aucuparia; así como Rhamnus alpina, Ribes alpinum, Viburnum lantana, Genista hispanica, Daphne laureola y Cytisus cantabricus. Es muy llamativa la ausencia total de Taxus baccata, al no contabilizarse siquiera un brinzal o chirpial, por lo que no hay reemplazo posible de los ejemplares adultos. Por el contrario, en los estratos inferiores sí se han herborizado Arabis alpina, Asplenium adiantum-nigrum, Brachypodium pinnatum subsp. rupestre, Echium vulgare, Helianthemum nummularium, Pentaglottis sempervirens, Polypodium vulgare, Primula veris subsp. columnae, Sideritis hyssopifolia y Teucrium pyrenaicum, entre otras. En la orla rupícola y arbustiva abierta de esta pequeña formación nemoral se desarrollan bastantes tejos viejos ramoneados e incluso en su forma tapizante coincidiendo con una mayor accesibilidad para el ganado.

El bosque mixto de L'Abiduriu se encuentra en la situación opuesta, toda vez que el estado general de los tejos es aceptable y la dinámica progresiva, pues hay algunos de porte subarbustivo 
cobijados en el interior de acebos. Además, se han encontrado numerosos brinzales de apenas $10 \mathrm{~cm}$ en las fisuras de las rocas y en cubiertas herbáceas nemorales densas. El núcleo arbóreo de esta comunidad tiene una mayor extensión superficial y una orla de tejos arbustivos y tapizantes dispersos a su alrededor. La cubierta arbórea principal, con alturas por encima de los 15 m, está dominada por Taxus baccata con ejemplares muy longevos. Algunos individuos singulares llegan a superar los 3 metros de perímetro de tronco (Figura 5). En el resto de los estratos, Ilex aquifolium está bien representado, mientras que el tejo aparece de forma testimonial junto a Fagus sylvatica, Sorbus aria, Crataegus monogyna y Corylus avellana.

\section{Figura 5. Tejos de L'Abiduriu}

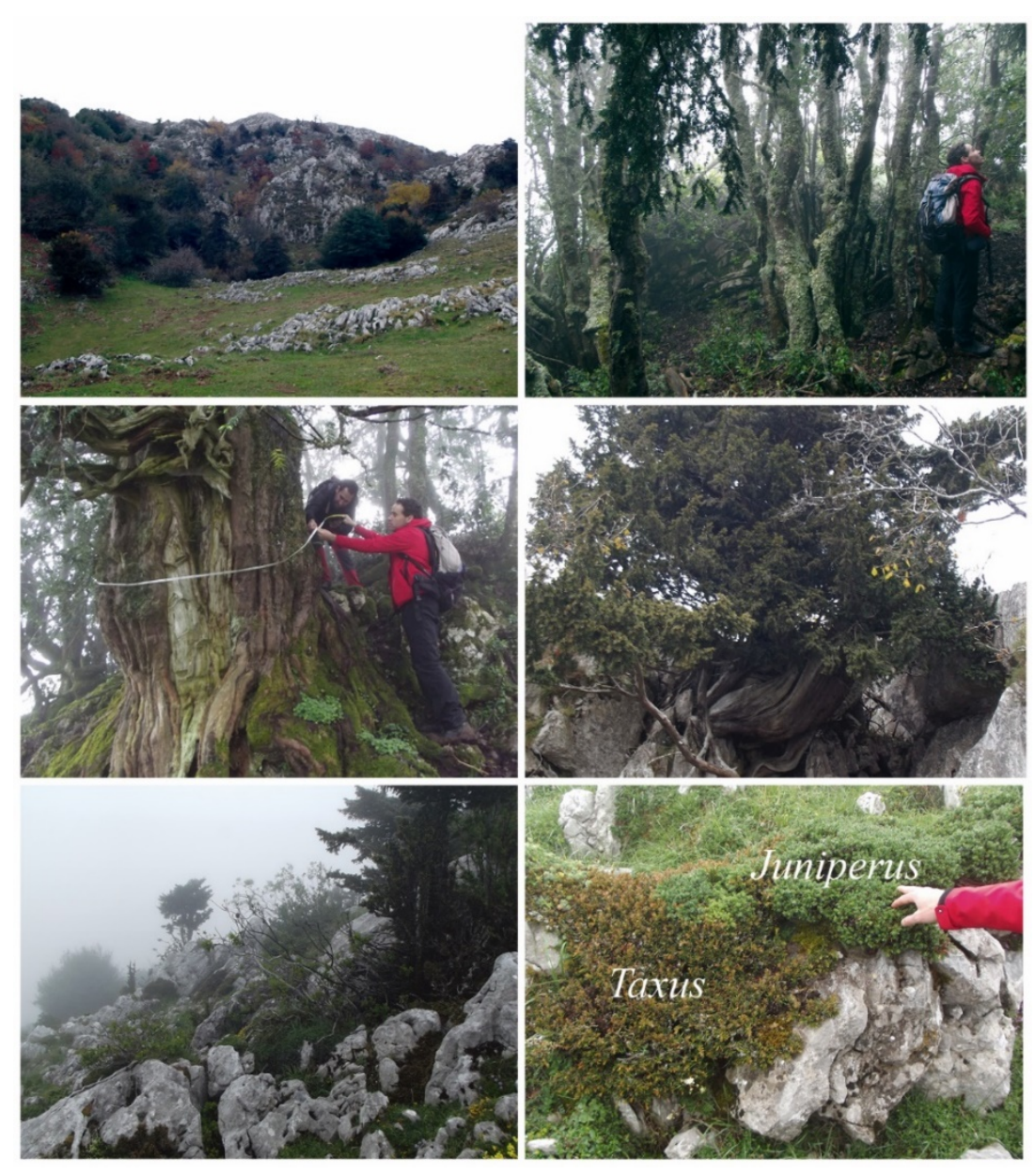

Fuente: elaboración propia

La presencia del haya y el acebo junto a otras herbáceas y arbustivas típicas de los bosques y los suelos ricos en bases que encontramos en los estratos inferiores -Daphne laureola, Helleborus viridis subsp. occidentalis, Rhamnus alpina, Carex brevicollis, Hepatica nobilis-, acercan fitosociológicamente esta comunidad a los hayedos eutrofos. De hecho, en algunos doseles el dominio arbóreo lo ejercen las hayas o incluso los acebos. Por tanto, se trata de bosques mixtos de tejo, acebo y haya con un cortejo florístico muy similar al de los hayedos -o bosques mixtos de 
hayas, mostajos y tejos - que se conservan en la parte alta de las laderas. En este sentido, se puede inferir que constituye la fase senescente del hayedo o una etapa de sustitución avanzada. En toda el área de L'Abiduriu, se aprecian rodales dispersos de tejos de porte arborescente entre los que se intercalan otros de acebo y afloramientos rocosos con tejos rastreros que cubren la roca, en los cuales se han encontrado varios ejemplares secos y extremadamente ramoneados.

\subsection{Primer acercamiento pedoantracológico}

Las labores de identificación y pesado muestran unos resultados interesantes: de ARAMO 1 cabe señalar la escasa antracomasa general $-28,77 \mathrm{mg} / \mathrm{kg}$-, especialmente en los niveles inferiores donde apenas se han encontrado carbones, tal y como cabía esperar por su condición de depósito antiguo de arcillas de descalcificación. Aun así, se han hallado algunos fragmentos que no han podido ser identificados con precisión por su mal estado, vitrificación y nimio tamaño. Es el mismo caso de los niveles superiores, aunque sí se consiguieron algunas identificaciones (Figura 6). En efecto, la mayor parte de las piezas de carbón se corresponden con pequeñas ramitas muy vitrificadas, en las que apenas había estructuras celulares que analizar entre la corteza y la médula. Por esto, en la mayoría de los casos el plano transversal se encontraba fundido, no se podían apreciar radios y únicamente se constataba la existencia de vasos - poros en el transversal- y estructuras propias de las angiospermas.

Así mismo, cabe subrayar el hallazgo de carbones de leñosas que hoy en día no se encuentran a esta altitud en el Aramo ni en las cercanías del punto de sondeo. En concreto, se recopilaron 3 fragmentos de Fagus sp, 4 de llex sp, 2 de Corylus sp y 12 correspondientes a Ericaceae. En los roquedos, a unos 100-150 m por debajo del área analizada (1550-1600 de altitud), sí aparecen ejemplares arbustivos de Juniperus alpina y Taxus baccata de los que se han recuperado 3 piezas de cada taxón además de otro de una conífera sin determinar. Pero sin duda, lo más llamativo ha sido la aparición de 158 fragmentos $(0,454$ gr) de angiospermas sin determinar, que en su mayoría se corresponden con ramas de tamaño nimio y otras demasiado vitrificadas para concretar más la identificación.

En cuanto a ARAMO 2, los resultados han sido más fructíferos por la cantidad de carbones, su buen estado y el peso de algunos fragmentos. Si bien es cierto que la antracomasa es de nuevo muy pobre $(23,75 \mathrm{mg} / \mathrm{kg})$, no obstante, los dos niveles más superficiales concentran la mayor parte de las muestras $(0,72 \mathrm{gr}$ de los 0,8 totales). Aunque el número de angiospermas - sin especificar - es alto (83 piezas con un peso conjunto de 0,2 gr), el taxón que predomina entre las identificaciones es Taxus baccata con un peso total de 0,21 gr y presente en todos los niveles del sondeo, incluso en los inferiores donde apenas se encontraron carbones y son todos monoespecíficos. 
Figura 6. Fragmentos de carbones al microscopio.

1 y 2) Taxus baccata. 3 y 4) Salix sp. 5 y 6) Betula sp.
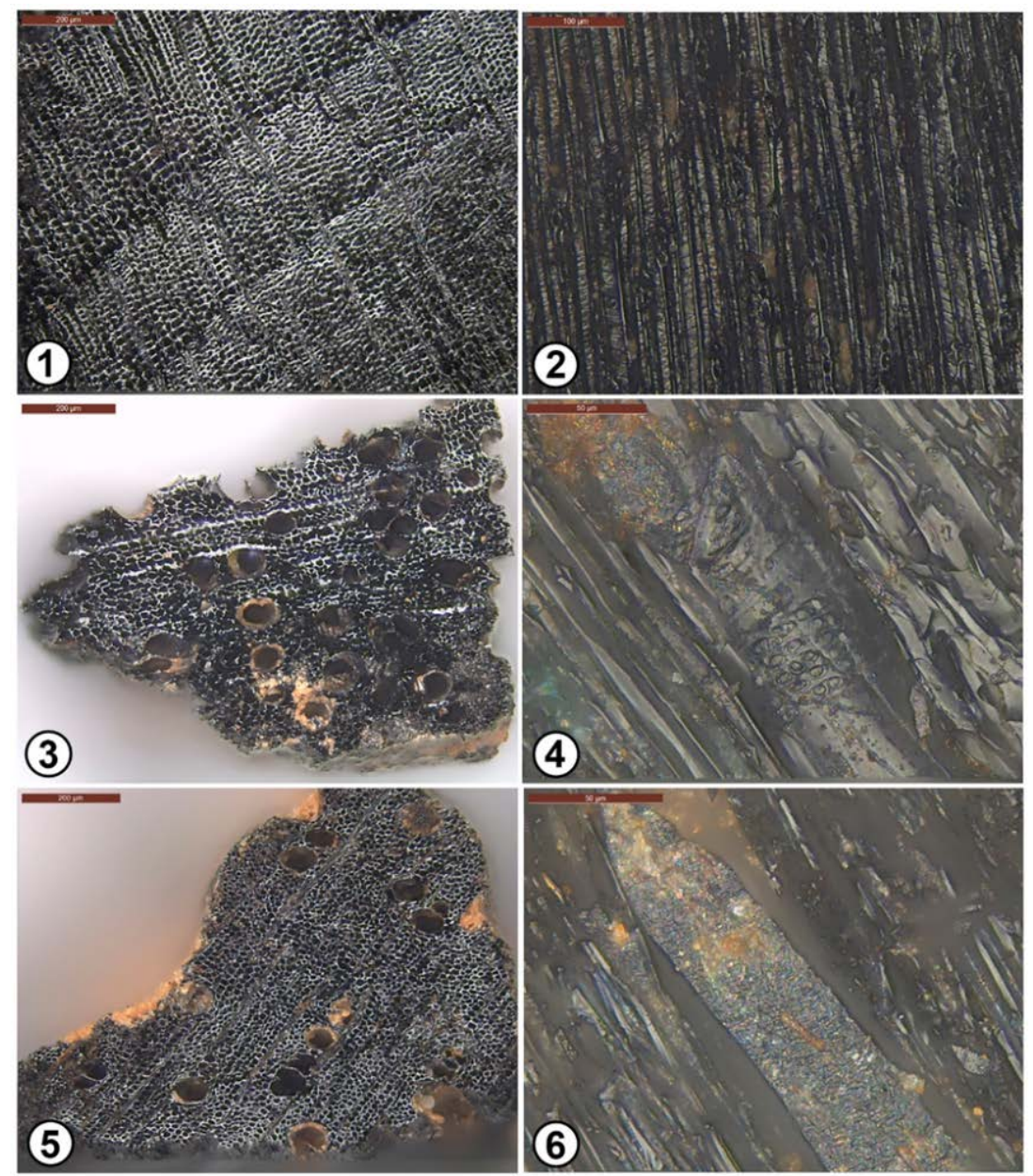

Fuente: elaboración propia

Otro hecho significativo es la mayor diversidad taxonómica y de elementos potencialmente arbóreos como Salix sp, Betula sp, Quercus - planocaducifolio- y Fraxinus sp. En la actualidad, ninguno de estos ni otros taxones alcanza una altitud superior a los $1600 \mathrm{~m}$ en todo el Aramo. En cuanto a las arbustivas: Corylus sp, Prunus sp, Ericaceae y Leguminosae alcanzan cotas cercanas pero inferiores y no cerca del punto de sondeo. Sólo Berberis fue identificada en un roquedo cercano.

Por otra parte, si bien ARAMO 1 presenta una mayor pobreza como se corresponde a unas condiciones más duras para las plantas debido a la altitud, ARAMO 2 -aun así, por encima de los $1600 \mathrm{~m}$ - muestra una gran biodiversidad. Es revelador en sendos casos la presencia también de Corylus sp, Ilex sp, Juniperus sp, ericáceas y, especialmente, de Taxus baccata.

Las dataciones de los carbones mediante AMS han quedado restringidas, debido al escaso peso de los fragmentos y a las limitaciones económicas, a unas pocas muestras, todas ellas correspondientes a especies arbóreas. 6 de las 7 muestras tienen una edad entre 3355 y 3450 cal BP y la restante es subactual (Figura 7). 
Esta última, única muestra que se encuentra fuera del rango temporal correspondiente a la Edad del Bronce es un fragmento de Fagus que, por desgracia, fue el único carbón de los hallados en ARAMO 1 con peso suficiente para ser datado. A riesgo de ser reiterativos, es importante subrayar que el haya no llega ni siquiera en forma arbustiva a estas cotas y, sin embargo, en algún momento reciente sí lo ha hecho. En lo que respecta a ARAMO 2, que concentra el grueso de las dataciones, se constata la migración de partículas dentro del suelo, máxime cuando fragmentos de la misma fecha aparecen con una distancia de $60 \mathrm{~cm}$ de profundidad y unos más antiguos aparecen sobre otros más recientes. Sea como fuere, sabemos que, en plena Edad del Bronce, hubo uno o varios incendios que calcinaron la cubierta forestal del punto muestreado. En concreto, se trataba de un bosque mixto de tejos y planocaducifolios similar a la comunidad relicta de Vatsongo, en la que tampoco se desarrollan actualmente hayas.

Figura 7. Resumen gráfico de los resultados pedoantracológicos

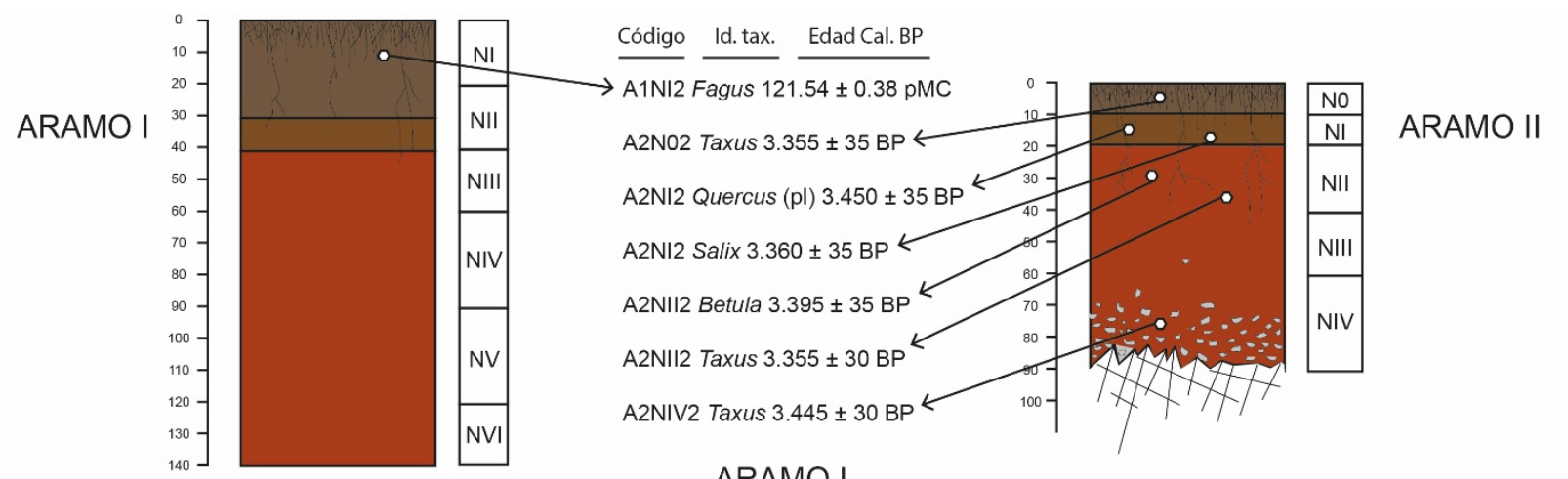

ARAMO I

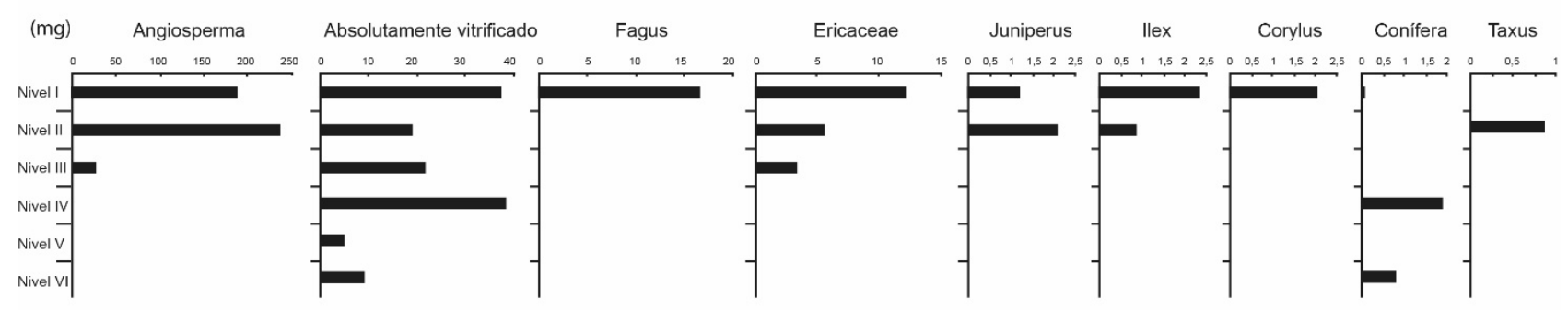

ARAMO ॥

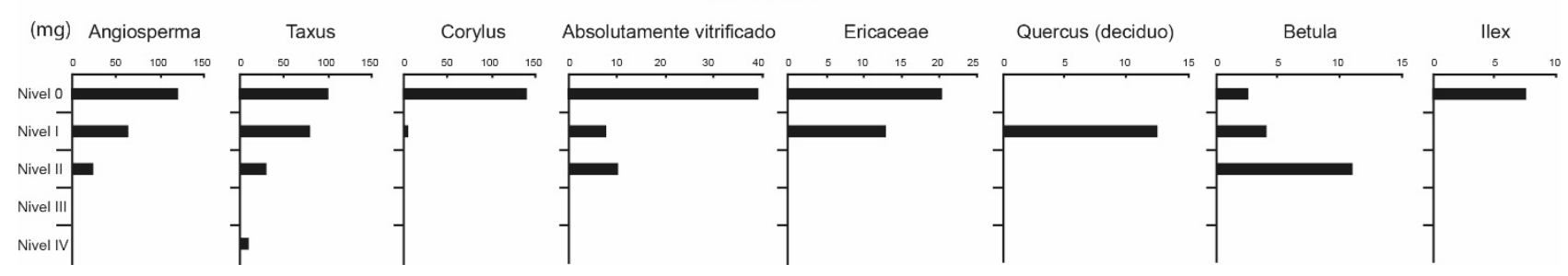

Fuente: elaboración propia. Las fechas de radiocarbono se han calibrado utilizando el programa OXCAL 4.3 (Bronk, 2017) y la curva de calibración IntCal13 (Reimer et al., 2013)

\section{Discusión}

La distribución actual y la evolución paleoecológica de Taxus baccata en el Macizo Asturiano es desconocida en toda su dimensión. A pesar de que es considerada una especie relicta en la 
Cordillera Cantábrica, propia de climas más fríos que el actual, no ha aparecido en los diagramas polínicos asturianos hasta la fecha (véase López-Merino, 2009). Sin embargo, el tejo ha tenido que participar en el paisaje vegetal del pasado, tal y como parece indicar nuestro hallazgo y se señala en otros trabajos cantábricos como los de Pérez-Díaz et. al (2013) y Uzquiano et. al (2015). Respecto a la Sierra del Aramo, hay escasos datos paleobotánicos: Dupré (1985, 1986 y 1988) aporta información extraída de su estudio polínico del túmulo de Mata'l Casare, a 1.300 m de altitud en el entorno del área megalítica de La Cobertoria, en la estribación meridional del Aramo. Se trata de un yacimiento calcolítico ( $V$ milenio BP) en el que el taxón mayoritario es Quercus (planocaducifolio) seguido de Pinus sylvestris tipo. También es relevante la constancia de Betula y Corylus, y en menor medida de Tilia, Alnus, Ulmus, Fagus e Ilex. En cualquier caso, no hay registro de tejo. Tampoco en el material leñoso proveniente de la quema en una hoguera de la necrópolis de La Cobertoria, fechado en el IV milenio BP, en la cual abunda el roble (Quercus robur), con un $62 \%$ del total, seguido por un $16 \%$ de encina (Quercus llex) y con avellano y otras angiospermas no identificadas (Blas, 2012).

Por el contrario, Blas et. al (2013) y Blas y Rodríguez (2015) obtienen una información relevante de los carbones vegetales pertenecientes a una de las cubetas de La Campa les Mines (cubeta 6), en el Sur de la vertiente oriental del Aramo. Con una cronología datada en el IV milenio BP, las maderas empleadas para la combustión fueron en su mayoría de Taxus, seguida por Quercus (planocaducifolio), Corylus, Betula e llex. Esto coincide también con los restos antracológicos procedentes de la propia mina de Texeu -en la que se extraían los materiales trabajados posteriormente en dicha campa- con actividad reconocida en la misma época. Todos los restos vegetales analizados procedentes de las galerías corresponden a Taxus, que se empleaba como leña para el arranque del mineral por el sistema de fire-setting y como teas de iluminación (Blas, 2014). Durante la explotación de aproximadamente un milenio de las minas, se estima que 4100 tejos fueron cortados y utilizados tanto para atacar la piedra con el fuego como para hacer teas y herramientas (Blas, 2014). Por tanto, la extensión de esta conífera tuvo que ser mucho mayor que en la actualidad, tal y como lo demuestra la persistencia de topónimos alusivos al tejo en toda la sierra, como el propio nombre de las minas.

Parece, por tanto, que el tejo ha estado vinculado al Aramo desde al menos la Edad del Bronce, y seguramente haya tenido una importante extensión en el Macizo Asturiano. No obstante, el hecho de que ningún análisis palinológico asturiano haga mención de este taxón nos lleva a pensar que el polen de Taxus no queda representado en los diagramas polínicos por alguna razón relacionada con sus propias características. En efecto, no es un taxón muy común en los registros polínicos debido a la fina y ornamentada capa exterior de su polen -exina- y a su vulnerabilidad a la corrosión (Havinga, 1964 y 1967; Moore et al., 1991; Beug, 2004; Pérez-Díaz et al., 2013). 
Sin embargo, Taxus baccata se encuentra hoy en numerosas zonas del Macizo Asturiano. Destacan, las poblaciones de Peña Gradura (pico Caldoveiro), Peñamayor, Freisdemora en la Sierra de Sobia y la consabida tejeda de la Sierra del Sueve, todos estos, enclaves calizos de mediana altitud. Otras áreas son Cuevas (Belmonte de Miranda), Sames (Amieva) y Perlunes (Somiedo) pero sólo los tejos del Sueve están considerados propiamente como tejeda y están protegidos por la legislación regional como comunidad. En el caso de la Sierra del Aramo, ni su situación como Paisaje Protegido sin declarar legalmente ni la del tejo, clasificado como especie de interés especial por el Gobierno del Principado de Asturias, han favorecido la conservación de este patrimonio natural y cultural. De hecho, la reintroducción del rebeco para surtir de piezas a la caza en la sierra ha sido un éxito y cuenta ya con unos 300 ejemplares (La Nueva España, 13/06/2017), lo que ha incrementado el ramoneo del tejo que ya realizaban otros ungulados salvajes y domésticos. Además, hay que añadir otro grave problema como es la extracción de individuos de Taxus baccata en el Aramo para la confección y comercialización de bonsáis, denunciada recientemente por naturalistas y el SEPRONA (La Nueva España, 27/08/2017).

Por otro lado, los problemas de regeneración del tejo han sido detectados en diversos enclaves europeos de las Islas Británicas (Tittensor, 1980; Hulme, 1996), Eslovenia (Dovciak, 2002), Dinamarca (Svenning \& Magård, 1999), Noruega (Mysterud \& Østbye, 2004) y España, donde han sido descritos, entre otros, por García et. al (2000 y 2015), García y Obeso (2003), García (2006) y Lavabre y García (2015), incluso de forma particular sobre la Sierra del Aramo. García (2006) indica el herbivorismo por ungulados como factor más limitante y, por el contrario, la importancia para la regeneración del tejo de las aves dispersantes de semillas y los arbustos que le ofrecen protección como plantas nodrizas. Beato et. al (2018) señalan como medida de conservación para los tejos del Aramo su inclusión dentro del hábitat de interés comunitario 9580 de Bosques mediterráneos de Taxus baccata y la declaración de esta unidad montana como Lugar de Interés Comunitario (LIC). Además, siguiendo a García \& Zamora (2003) es indispensable la catalogación y protección estricta, por lo menos, de los ejemplares más longevos para garantizar el futuro de la especie y los ecosistemas en que participa regionalmente.

Efectivamente, los planes de gestión del Gobierno del Principado de Asturias son insuficientes. En este sentido, el Decreto 145/2001, de 13 de diciembre, sanciona el Plan de Manejo del Tejo (Taxus baccata) como especie protegida. Sigue criterios relacionados con el desarrollo sostenible -establecer y aplicar medidas de protección, promover la explotación racional- y persigue evitar las amenazas que se ciernen sobre las especies catalogadas para conseguir un estado de conservación estable. No obstante, en este caso llama la atención la descripción de unos problemas de conservación escasos, reducidos a la afección por posibles malos tratos a los ejemplares más longevos y de porte notable. Se desconoce o subestima, por tanto, el robo para el tráfico de bonsáis y el intenso ramoneo al que someten los animales domésticos y salvajes a los 
tejos. Así pues, es necesario, por un lado, un mejor conocimiento sobre el tejo y las formaciones vegetales en las que participa en el Macizo Asturiano (su estado y dinámica); por otro, medidas de protección específicas que garanticen el mantenimiento de algunas de estas comunidades singulares, como las de L'Abeduriu y Vatsongo en el Aramo. A su conservación podría contribuir, por ejemplo, su declaración como Monumento Natural o su inclusión en una Zona de Especial Conservación para el Aramo en su conjunto. Queda por ver, además, el futuro del tejo en un contexto de cambio global, de aumento de las temperaturas y fuertes transformaciones tanto ecológicas como socioeconómicas.

Por último, la aplicación del método pedoantracológico es muy adecuado para los estudios de carácter local y, especialmente, para lugares donde es difícil aplicar otras técnicas de análisis paleoecológicas y reunir datos sobre taxones que estas pueden ignorar. Uno de los resultados de mayor utilidad es el del valor de la antracomasa, tan necesario para realizar comparaciones y establecer patrones de evolución más generales. En el Aramo, un hecho muy notable es la baja antracomasa. La relación entre el peso de los fragmentos de carbón y el suelo extraído seco muestra cifras similares a las obtenidas en estudios pedoantracológicos en el límite superior del bosque en los Pirineos, a mucha mayor altitud. Cunill (2010) y Cunill et al. (2012 y 2013) examinaron antracomasas en torno a los $100 \mathrm{mg} / \mathrm{kg}$ entre los $1995 \mathrm{~m}$ y los $2200 \mathrm{~m}$ de altitud, así como cercanas a los $10 \mathrm{mg} / \mathrm{kg}$ desde los $2300 \mathrm{~m}$ hasta los 2500. Nada que ver con los resultados de montañas mediterráneas como la Sierra de Gredos, donde a 1700 m García Álvarez et al. (2017) han calculado 23487,03 mg/kg y a 2200 m 2678,31 mg/kg; ni con los máximos de fosas pedoantracológicas de espacios montanos más xéricos y a menor altitud (1300-1400 m) como Sierra Bermeja donde un solo nivel sobrepasó los 74000 mg/kg (Gómez-Zotano et al., 2017; Olmedo-Cobo et al., 2017). Obviamente, esto puede estar relacionado con un escaso número de incendios o unas circunstancias locales peculiares para la conservación de los carbones. Sin embargo, no hay estudios recientes que indiquen este tipo de suelos, litologías y usos antrópicos especialmente dañinos para la conservación de los carbones. A la espera de nuevas prospecciones en el Aramo, la hipótesis más razonable es la de escasos eventos de fuego, lo cual contrasta con el elevado número de incendios que se dan en la región en la actualidad, aunque bien es cierto que, en una situación de abandono del medio rural, menor carga ganadera y a cotas más bajas donde la vegetación leñosa prospera con rapidez.

En definitiva, ha sido un hallazgo muy elocuente y relevante encontrar en ámbitos absolutamente deforestados en la actualidad, utilizados como pastizales desde tiempos inmemoriales, carbones correspondientes a especies potencialmente arbóreas. Por otro lado, la cronología aportada por las dataciones coincide con los datos arqueológicos obtenidos en el Aramo (Blas, 2012, 2013, 2014; Blas y Rodríguez, 2015), que indican la Edad del Bronce como un periodo de intensa ocupación humana, de colonización de las cumbres y de desarrollo de diversas actividades de origen 
neolítico. En este sentido, es muy expresivo que 6 de las 7 muestras datadas tengan una edad muy similar.

\section{Conclusiones}

Taxus baccata participa en diferentes asociaciones vegetales del Macizo Asturiano con algunas poblaciones realmente importantes y variadas como las de la Sierra del Aramo. El bosque de Vatsongo presenta una composición florística que no es atribuible a ninguna de las asociaciones vegetales descritas hasta la fecha y constituye un patrimonio excepcional; igualmente los tejos de L'Abiduriu con magníficos ejemplares dignos de una atención y cuidado especial. Por otro lado, las formaciones arbustivas de matorral petrano presentan una amplitud florística mayor de la descrita fitosociológicamente y un estado más controvertido por las actividades antrópicas.

En cuanto a la evolución holocena del taxón analizado, la aplicación del método pedoantracológico, por vez primera en el Macizo Asturiano, revela que Taxus baccata ha estado presente en el paisaje al menos desde hace 3450 años. Las dataciones de fragmentos de carbón de tejos y varias planocaducifolias potencialmente arbóreas indican incendios en la Edad del Bronce, coincidiendo con la mayor presencia e impacto por la colonización humana de las montañas del norte peninsular. En efecto, los paisajes abiertos de la plataforma culminante del Aramo son fruto de cambios milenarios en el paisaje en momentos de intervención y explotación antrópica que han mantenido el límite superior del bosque muy por debajo del cinturón subalpino pero, del mismo modo, han preservado dos manchas con tejos realmente singulares.

La conservación futura de estas comunidades está en duda a pesar de la protección regional de Taxus baccata y las directrices europeas para algunos de los hábitats en que participa. Ciertamente, diversas amenazas se ciernen sobre el tejo, con problemas de regeneración analizados en todo Europa y afectado obviamente por los procesos ligados al cambio global. Por tanto, son necesarias medidas más eficaces de protección y estudios precisos sobre la dinámica de la especie y su respuesta adaptativa a los nuevos contextos ecológicos y socioeconómicos.

Agradecimientos: Este trabajo no hubiera sido posible sin la inestimable ayuda de José Luis Marino Alfonso, José Manuel Rodríguez Berdasco, Adriano Álvarez Méndez, Manuel Rodríguez Rastrero, Ignacio Abella Mina, Luis Carlón, la familia Cobos (Toulouse-Otívar), la familia Fernández-Conde de Las Casas del Puente (Salas), Héctor Rato Martín, Sergio Rato, Ícaro Obeso Muñiz, Marco de la Rasilla, Marta Alonso Guervós, Francisco Javier Alonso Rodríguez y Ángel Rodríguez Rey. Asimismo, queremos dar la gracias por su apoyo a i-Ge\&ser (Oviedo), al GRAMP (Barcelona) y al GEODE (Toulouse), especialmente a Vanessa Py-Saragaglia Jean Paul Métailié y Didier Galop. S. Beato agradece también a la Fundación Alvargonzález de Gijón la concesión de la XXVI Ayuda de 
investigación Ramón de la Sagra para completar el proyecto "Dinámica paleoecológica del Macizo Central Asturiano a través de un transecto pedoantracológico".

Declaración responsable: Las/os autoras/es declaran que no existe ningún conflicto de interés con relación a la publicación de este artículo. Las/os autoras/es declaran que no existe ningún conflicto de interés con relación a la publicación de este artículo. Todos han participado en la elaboración y corrección de los textos, gráficos y mapas. S. Beato y R. Cunill realizaron el análisis pedoantracológico. 


\section{Bibliografía}

Abella, I. (2007). La Memoria del bosque. Crónicas de la vieja selva europea. Cultos y culturas, mitos, leyendas y tradiciones. Barcelona: Integral RBA Libros SA.

Abella, I. (2009a). La Cultura del Tejo. Esplendor y decadencia de un patrimonio vital. Valladolid: Urueña SL.

Abella, I. (2009b). Tejedas del sueve (informe para su gestión). Efecto antrópico y dinámica de un ecosistema singular. Las viescas del sueve. Gobierno del Principado de Asturias.

Álvarez, A., Caballero, D., Marino, J. L., \& Rodríguez, J. M. (2006). La Tejeda del Sueve: un problema de regeneración natural. Comunicación presentada en el III Congreso Español de Biogeografía (Unpublished).

Arozena, M. E., \& Molina, P. (2000). Estructura de la vegetación. In G. Meaza (Dir.), Metodología y práctica de la Biogeografía (pp. 77-147). Barcelona: Serbal.

Beato, S. (2012). El paisaje vegetal de la Sierra del Aramo, un medio de montaña en transformación (Montaña Central de Asturias). In R. Cunill, A. Pèlachs, R. Pérez-Olbiol \& J.M. Soriano (Coords.), Las zonas de montaña: gestión y diversidad (pp. 321-335). Barcelona: Departament de Geografía de la Universitat Autònoma de Barcelona.

Beato, S. (2018). El patrimonio natural de la Sierra del Aramo (Montaña Central Asturiana) y la evolución de su paisaje (Doctoral dissertation, Universidad de Oviedo, Spain) (Unpublished).

Beato, S., Poblete, M. Á., \& Marino, J. L. (2016a). Hábitats forestales de interés comunitario en la Sierra del Aramo (Montaña Central Asturiana, España), lugar de encuentro entre dos distritos biogeográficos contrastados. In J. Gómez Zotano, J. Arias García, J.A. Olmedo Cobo \& J.L. Serrano Montes, (Eds.), Avances en Biogeografía. Áreas de distribución: entre puentes y barreras (pp. 494-502). Granada: Ediciones de la Universidad de Granada, Tundra.

Beato, S., Poblete, M. Á., \& Marino, J. L. (2016b). La expansión del matorral y su caracterización biogeográfica en la Sierra del Aramo (Montaña Central Asturiana, España). In J. Gómez Zotano, J. Arias García, J.A. Olmedo Cobo \& J.L. Serrano Montes (Eds.), Avances en Biogeografía. Áreas de distribución: entre puentes y barreras (pp. 494-502). Granada: Ediciones de la Universidad de Granada, Tundra.

Beato, S., Marino, J.L., \& Poblete, M.Á. (2017). El paisaje vegetal y los hábitats forestales de interés comunitario en la Montaña Central Asturiana. Cuadernos Geográficos, 56(1), $26-52$. Retrieved from http://revistaseug.ugr.es/index.php/cuadgeo/article/view/4834

Beato, S., Álvarez, A., Marino, J.L., Poblete, M.A., \& Rodríguez, J.M. (2018). Nuevas aportaciones para la inclusión de los tejos de la Sierra del Aramo (Macizo Central Asturiano) en el hábitat 9580 
de bosques mediterráneos de Taxus baccata L. In R. U. Gosálvez, M. C. Díaz, J. L. García, M. A. Serrano de la Cruz \& Ó. Jerez (Coords.), Bosque mediterráneo y humedales: paisaje, evolución y conservación. Aportaciones desde la Biogeografía (pp. 567-577). Ciudad Real: Almud Ediciones de Castilla-La Mancha.

Benham, S. E., Houston Durrant, T., Caudullo, G., \& de Rigo, D. (2016). Taxus baccata in Europe: distribution, habitat, usage and threats. In J. San-Miguel-Ayanz, D. de Rigo, G. Caudullo, T. Houston Durrant \& A. Mauri (Eds.), European Atlas of Forest Tree Species (pp. e015921+). Luxembourg: Publ. Off. EU. Retrieved from https://forest.jrc.ec.europa.eu/media/atlas/Taxus_baccata.pdf

Bertrand, G. (1966). Pour une étude géographique de la végétation. Revue Géographique des Pyrénées et du Sud-Ouest, T. XXXVII, Fasc. 2, 129-143.

Beug, H.J. (2004). Leiffaden der Pollenbestimmung für Mitteleuropa und angrenzende Gebiete. Munich: Pfeil.

Blanco, E., Vasco, F., Abellà, I., \& Cortés, S. (2008). Tejo y cultura: de la tradición etnobotánica a la farmacología científica. In A. Caritat (Dir.), II Jornadas sobre el tejo en el Mediterráneo Occidental (pp. 11-34). Girona.

Blas, M. Á. de (2012). Beneficio y consagración de las cumbres: el caso de "Los Fitos" y la concurrencia Neolítico-Bronce antiguo en el área megalítica de La Cobertoria, Asturias. In J. R. Muñiz (Coord.): Ad orientem. Del final del paleolítico en el norte de España a las primeras civilizaciones del Oriente próximo. Estudios en homenaje al profesor Juan Antonio Fernández Tresguerres Velasco (pp. 307-347). Oviedo: Ediciones de la Universidad de Oviedo, Ménsula Ediciones.

Blas, M. Á. de (2013). IV milenio a. de C.: los monumentos sepulcrales del Puerto de la Cobertoria (Quirós) y el dominio de las cumbres por las sociedades neolíticas. In M. Á. de Blas (Coord..), De neandertales a albiones: cuatro lugares esenciales de la Prehistoria en Asturias (pp. 69-138). Oviedo: Real Instituto de Estudios Asturianos.

Blas, M. Á. de (2014). El laboreo del cobre en la Sierra del Aramo (Asturias) como referente cardinal de la minería prehistórica en la región cantábrica. Cuadernos de Prehistoria y Arqueología, 24, 45-84.

Blas, M. Á. de, \& Rodríguez, F. (2015). La cuestión campaniforme en el Cantábrico central y las minas de cobre prehistóricas de la sierra del Aramo. Cuadernos de Prehistoria y Arqueología, 41, 165-179.

Braun-Blanquet, J. (1979). Fitosociología. Bases para el estudio de las comunidades vegetales. Madrid: Blume. 
Burgarella, C., Berganzo, E., Zabala, M., Prada, A., Iglesias, A., Riba, M., Mayol, M., Vendramin, S., \& González, G. (2010). Aspectos genéticos y demográficos de Taxus baccata en la Red de Parques Nacionales. In L. Ramírez, \& B. Asensio (Eds.), Proyectos de investigación en la red de Parques Nacionales 2007-2010 (pp. 125-137). Madrid.

Camprodon, J., Guixé, D., Casals, P., Caritat, A., Buqueras, X., García-Martí, X., ... Casas C. (2016). Conservation of Mediterranean yew forests. Best practice handbook. Life TAXUS Project. Forest Science Centre of Catalonia. Retrieved from http://aifm.org/sites/default/files/fichiers/manual_taxus_ang.compressed_0.pdf

Carlo, T. A., García, D., Martínez, D., Gleditsch, J., \& Morales, J. M. (2013). Where do seeds go when they go far? Distance and directionality of avian seed dispersal in heterogeneous landscapes. Ecology, 94(2), 301-307. https://doi.org/10.1890/12-0913.1

Cortés, S., Vasco, F., \& Blanco, E. (2000). El libro del tejo (Taxus baccata L.). Madrid: ARBA.

Costa, M., Morla, C., \& Sainz, H. (Eds.) (1998). Los bosques ibéricos. Una interpretación geobotánica. Madrid: Geoplaneta.

Cunill, R. (2010). Estudi interdisciplinari de l'evolució del límit superior del bosc durant el període holocènic a la zona de Plaus de Boldís-Montarenyo, Pirineu central català. Pedoantracologia, palinologia, carbons sedimentaris i fonts documentals (Doctoral dissertation, Universitat Autònoma de Barcelona, Spain) (Unpublished).

Cunill, R., Soriano, J.M., Bal, M.C., Pèlachs, A., \& Pérez-Obiol, R. (2012). Holocene treeline changes on the south slope of the Pyrenees: a pedoanthracological analysis. Vegetation History and Archaeobotany, 21, 373-384. https://doi.org/10.1007/s00334-011-0342-y

Cunill, R., Soriano, J.M., Bal, M.C., Pèlachs, A., Rodriguez, J.M., \& Pérez-Obiol, R. (2013). Holocene high-altitude vegetation dynamics in the Pyrenees: a pedoanthracology contribution to an interdisciplinary approach. Quaternary International, 289, 6070. https://doi.org/10.1016/j.quaint.2012.04.041

Díaz, T. E. (2010). Tejos y tejadas: un patrimonio natural y cultural que debemos conservar y proteger. Peña Santa: Revista del Grupo de Montaña Peña Santa, 6, 72-82.

Dovciak, M. (2002). Population dynamics of the endangered English yew (Taxus baccata L.) and its management implications for biosphere reserves of the western Carpathians. Division of Ecological Sciences, MAB Secretariat, UNESCO.

Dupré, M. (1985). Contribución del análisis polínico al conocimiento del paleoambiente en España (Doctoral dissertation, Universidad de Valencia, Spain) (Unpublished). 
Dupré, M. (1986). Contribution de l'analyse pollinique a la connaissance du paléoenvironnement en Espagne. L'Anthropologie, 90 (3), 589-591.

Dupré, M. (1988): Palinología y paleoambiente. Nuevos datos españoles. Referencias. Trabajos Varios del S.I.P., 84. Valencia: Diputación provincial de Valencia.

Fidalgo, C. (1997). El trabajo de campo en Biogeografía. Didáctica geográfica, 2, 33-44.

García, D., Zamora, R., Hódar, J.A., Gómez, J.M., \& Castro, J. (2000). Yew (Taxus baccata L.) regeneration is facilitated by fleshy-fruited shrubs in Mediterranean environments. Biological Conservation, 95, 31-38. https://doi.org/10.1016/S0006-3207(00)00016-1

García, D., \& Obeso, J.R. (2003). Facilitation by herbivore-mediated nurse plants in a threatened tree Taxus baccata: local effects and landscape level consistency. Ecography, 26, 739750. https://doi.org/10.1111/i.0906-7590.2003.03601.x

García, D., \& Zamora, R. (2003). Persistence, multiple demographic strategies and conservation in long-lived Mediterranean plants. Journal of Vegetation Science, 14, 921926. https://doi.org/10.1111/j.1654-1103.2003.tb02227.x

García, D. (2006). Regeneración natural y conservación del tejo Taxus baccata L. en la cordillera Cantábrica: la importancia de las interacciones ecológicas. In L. Serra (Ed.), El Tejo en el Mediterráneo occidental (pp. 31-39). CAM.

García, D., Martínez, D., \& Lavabre, J.E. (2015). Regeneración del Tejo en las montañas cantábricas: ampliando el enfoque a través del espacio, el tiempo y la complejidad ecológica. In W.AA., Actas de las IV Jornadas Internacionales del Tejo (pp. 17-27). Lleida: CTFC.

García, S., Bal, M.C., Allée, P., García-Amorena, I., \& Rubiales, J. M. (2017). Holocene treeline history of a high-mountain landscape inferred from soil charcoal: The case of Sierra de Gredos (Iberian Central System, SW Europe). Quaternary International, 457, 8598. http://dx.doi.org/10.1016/j.quaint.2017.04.019

Gómez-Zotano, J., Olmedo-Cobo, J.A., Cunill-Artigas, R., \& Martínez-lbarra, E. (2017). Descubrimiento y caracterización geográfica de una depresión ultramáfica en Sierra Bermeja: nuevos datos geomorfoedáficos, fitogeográficos y paleoecológicos. Pirineos, 172, e026. http://dx.doi.org/10.3989/Pirineos.2017.172001

Havinga, A.J. (1964). 64 Investigations into the Differential Corrosion Susceptibility of Pollen and Spores. Pollen et Spores, 6, 621-635.

Havinga, A.J. (1967). Palynology and pollen preservation. Review of Palaeobotany and Palynology, 2(1-4), 81-98. 
Hulme, P.E. (1996). Natural Regeneration of Yew (Taxus Baccata L.): Microsite, Seed or Herbivore Limitation? Journal of Ecology, 84(6), 853-861. http://dx.doi.org/10.2307/2960557

Lavabre, J., \& García, D. (2015). Geographic consistency in the seed dispersal patterns of Taxus baccata $L$ in the Iberian Peninsula. Forest Systems, 24(3), e040. http://dx.doi.org/10.5424/fs/2015243-07462.

Lence, C., Molina, A., \& Acedo, C. (2011). Análisis del comportamiento fitosociológico del tejo (Taxus baccata L.) en el noroeste de la Península Ibérica. Spanish Journal of Rural Development, 7-22. http://dx.doi.org/10.5261/2011.ESP2.02

Linares, J.C. (2013). Shifting limiting factors for population dynamics and conservation status of the endangered English yew (Taxus baccata L., Taxaceae). Forest Ecology and Management, 291, 119-127. https://doi.org/10.1016/j.foreco.2012.11.009

López-Merino, L. (2009). Paleoambiente y antropización en Asturias durante el Holoceno. (Doctoral dissertation, Universidad Autónoma de Madrid, CSIC, Spain) (Unpublished).

Moore, P.D., Webb, J.A., \& Collinson, M.E. (1991). Pollen Analysis. Londres: Blackwell Sci. Publ. Mysterud, A., \& Østbye, E. (2004). Roe deer (Capreolus capreolus) browsing pressure affects yew (Taxus baccata) recruitment within nature reserves in Norway. Biological Conservation, 120, 545-548. http://dx.doi.org/10.1016/j.biocon.2004.03.027

Navarro, F. (1974a). Estudio de la flora y vegetación de la sierra del Aramo y sus estribaciones (Asturias) (Doctoral dissertation, Universidad de Oviedo, Spain) (Unpublished).

Navarro, F. (1974b). La vegetación de la Sierra del Aramo y sus estribaciones (Asturias). Revista Facultad Ciencias, 15(1), 111-243.

Navarro, F. (1976). Datos para el catálogo florístico del Aramo y sus estribaciones (Asturias). I: de Equisetaceae a Linaceae. Boletín del Instituto de Estudios Asturianos. Suplemento de ciencias, 22, 45-108.

Olmedo-Cobo, J.A., \& Gómez-Zotano, J. (2014). El tejo en el sur de España: análisis geoecológico y propuesta de conservación de una población mediterránea en peligro crítico de extinción. Bosque, 35(1), 23-36. http://dx.doi.org/10.4067/S0717-92002014000100003

Olmedo-Cobo, J.A., Cunill-Artigas, R., Martínez-lbarra, E. \& Gómez-Zotano, J. (2017). Paleoecología de Abies sp. en Sierra Bermeja (sur de la península ibérica) durante el Holoceno Medio a partir del análisis pedoantracológico. Bosque, 38 (2), 259270. http://dx.doi.org/10.4067/S0717-92002017000200004

Panareda, J.M. (1996). Cartografía de la Vegetación. Serie Geográfica, 6, 11-34. 
Pérez-Díaz, S., López-Sáez, J. A., Ruiz-Alonso, M., Zapata, L., \& Abel-Schaad, D. (2013). Holocene history of Taxus baccata in the Basque Mountains (Northern Iberian Peninsula). LAZAROA, 34, 29-41.

Rodríguez, M., Ramil-Rego, P., Díaz, R., Pereira-Espinel, J., \& Real, C. (2011). Los bosques dominados por Taxus baccata L. del extremo occidental de la Cordillera Cantábrica: caracterización, valor de conservación y amenazas. In J.M. Ninot (Ed.), Botànica PirenaicoCantàbrica. Actes del IX Colloqui Internacional de Botànica Pirenaico-Cantàbrica (pp. 367-378). CENMA.

Rodríguez Rastrero, M. (2015). Los suelos de Asturias (España): un enfoque basado en las relaciones entre factores formadores y horizontes de diagnóstico (Doctoral dissertation, Universidad Autónoma de Madrid, Spain) (Unpublished).

Ruiz de la Torre, J. (2006). Flora mayor. Madrid: Organismo Autónomo Parques Nacionales, Ministerio de Medio Ambiente.

Sanz, R., Pulido, F., \& Nogués-Bravo, V. (2009). Predicting mechanisms across scales: amplified effects of abiotic constraints on the recruitment of yew (Taxus baccata). Ecography, 32, 9931000. https://doi.org/10.1111/j.1600-0587.2009.05627.x

Schweingrüber, H.S. (1990a). Anatomie europäischer Hölzer. Stuttgart: W.S.L.F.N.P.

Schweingrüber, H.S. (1990b). Microscopic Wood Anatomy. Swiss Federal Institute for Forest, Snow and Landscape Research.

Serrá, L. (2009). 9580* Bosques mediterráneos de Taxus baccata (*). In W.AA., Bases ecológicas preliminares para la conservación de los tipos de hábitat de interés comunitario en España. Madrid: Ministerio de Medio Ambiente, y Medio Rural y Marino.

Svenning, J.CH., \& Magård, E. (1999). Population ecology and conservation status of the last natural population of English yew Taxus baccata in Denmark. Biological Conservation, 88, 173182. https://doi.org/10.1016/S0006-3207(98)00106-2

Thomas, P.A., \& Polwart, A. (2003). Biological flora of the British Isles. Taxus baccata L. Journal of Ecology, 91, 489-524.

Tittensor, R.M. (1980). Ecological history of yew Taxus baccata in southern England. Biological Conservation, 17, 243-265.

Uzquiano, P., Allué, E., Antolín, F., Burjachs, F., Picornell, LI, Piqué, R., \& Zapata, L. (2015). All about yew. On the trail of Taxus baccata in SW Europe by means of integrated palaeobotanical and archaeobotanical studies. Veg. Hist. Archaeobotany, 24, 229247. https://doi.org/10.1007/s00334-014-0475-x 
Vernet, J.L., Ogereau, P., Figueiral, I., Machado, C., \& Uzquiano, P. (2001). Guide d'identification des charbons de bois préhistoriques et récents. Sud-Ouest de l'Europe, France (Péninsule ibérique et Îles Canaries). Paris: CNRS Editions. 


\section{Anexo I. Inventarios de vegetación}

\section{Inventarios de vegetación en las áreas dominadas}

\section{por tejos, acebos y hayas de L'Abeduriu}

\begin{tabular}{|c|c|c|c|c|}
\hline & 7 & 2 & 3 \\
\hline 5 & (estrato arbóreo) & 5 & 3 & 0 \\
\hline & Corylus avellana $\mathrm{L}$ & 1 & - & - \\
\hline & Crataegus monogyna Jacq. & 1 & - & - \\
\hline & Fagus sylvatica $\mathrm{L}$. & - & 1 & - \\
\hline & Ilex aquifolium L. & 3 & 1 & - \\
\hline & Sorbus aria (L.) Crantz & 1 & - & - \\
\hline & Taxus baccata $\mathrm{L}$. & 3 & 4 & - \\
\hline 4 & (estrato arborescente) & 1 & 2 & 0 \\
\hline & Corylus avellana $\mathrm{L}$. & - & 1 & - \\
\hline & Ilex aquifolium L. & 4 & 4 & - \\
\hline & Sorbus aria (L.) Crantz & 3 & - & - \\
\hline & Taxus baccata L. & - & 2 & - \\
\hline 3 & (estrato arbustivo) & 1 & 2 & 0 \\
\hline & Corylus avellana $\mathrm{L}$. & - & 1 & - \\
\hline & Ilex aquifolium L. & 5 & 2 & - \\
\hline & Rhamnus alpina L. & - & 1 & - \\
\hline & Taxus baccata L. & - & 1 & - \\
\hline 2 & (estrato subarbustivo) & 2 & 1 & + \\
\hline & Brachypodium sylvaticum (Huds.) P. Beauv. & 1 & - & - \\
\hline & Daphne laureola L. s.I. & 3 & 1 & - \\
\hline & Fagus sylvatica $\mathrm{L}$. & 1 & - & - \\
\hline & Helleborus viridis subsp. occidentalis (Reut.) Schiffn. & 1 & 1 & - \\
\hline & Ilex aquifolium $\mathrm{L}$. & 3 & 2 & - \\
\hline & Rhamnus alpina L. & - & 1 & + \\
\hline & Taxus baccata L. & 1 & - & - \\
\hline 1 & (estrato herbáceo) & 4 & 4 & 5 \\
\hline & Aquilegia vulgaris subsp. vulgaris L. & 1 & - & - \\
\hline & Asphodelus albus Mill. & - & - & 1 \\
\hline & Asplenium trichomanes subsp. quadrivalens D.E. Mey. & - & - & 1 \\
\hline & Campanula arvatica subsp. Arvatica Lag. & - & 1 & 1 \\
\hline & Carex brevicollis DC. & 1 & 1 & - \\
\hline & Crataegus monogyna Jacq. & 1 & - & - \\
\hline & Daphne laureola L. s.I. & - & - & 1 \\
\hline & Dryopteris affinis (Lowe) Fraser-Jenk. & 1 & - & - \\
\hline & Euphorbia amygdaloides L. & 1 & - & - \\
\hline & Euphorbia flavicoma DC. & - & - & 3 \\
\hline & Globularia nudicaulis L. & - & - & 1 \\
\hline & Helianthemum nummularium (L.) Mill. & - & - & 1 \\
\hline & Helleborus viridis subsp. occidentalis (Reut.) & - & - & 1 \\
\hline & Hepatica nobilis Schreb. & 1 & 1 & 1 \\
\hline & Hippocrepis comosa L. & - & - & 4 \\
\hline & Ilex aquifolium L. & 1 & 1 & - \\
\hline & Mercurialis perennis L. & 1 & - & - \\
\hline & Paris quadrifolia $\mathrm{L}$. & - & 1 & - \\
\hline & Polygonatum verticillatum (L.) All. & - & 1 & - \\
\hline & Rhamnus alpina L. & 1 & 1 & - \\
\hline & Sanicula europaea L. & 1 & - & - \\
\hline & Saxifraga hirsuta subsp. hirsuta L. & 1 & - & - \\
\hline & Saxifraga paniculata Mill. & - & - & 3 \\
\hline & Scilla lilio-hyacinthus L. & 1 & - & - \\
\hline & Taxus baccata $\mathrm{L}$. & + & 1 & 1 \\
\hline & Teucrium pyrenaicum L. & - & - & 1 \\
\hline & Viburnum lantana L. & 1 & - & - \\
\hline
\end{tabular}




\section{Inventarios de vegetación en el bosque mixto de Vatsongo}

\begin{tabular}{|c|c|c|c|}
\hline & \\
\hline & & 1 & 2 \\
\hline \multirow[t]{7}{*}{5} & (estrato arbóreo) & 3 & 3 \\
\hline & Acer pseudoplatanus L. & 3 & 2 \\
\hline & Betula celtiberica Rothm. \& Vasc. & 3 & 2 \\
\hline & Sorbus intermedia (Ehrh.) Pers. & 2 & 2 \\
\hline & Taxus baccata $\mathrm{L}$. & 3 & 4 \\
\hline & Tilia platyphyllos Scop. & 3 & 2 \\
\hline & Ulmus glabra Huds. & 1 & 1 \\
\hline \multirow[t]{8}{*}{4} & (estrato arborescente) & 2 & 2 \\
\hline & Acer pseudoplatanus L. & 3 & 2 \\
\hline & Betula celtiberica Rothm. \& Vasc. & 2 & 2 \\
\hline & Corylus avellana $\mathrm{L}$. & 2 & 2 \\
\hline & Sorbus aria (L.) Crantz & 2 & 2 \\
\hline & Sorbus aucuparia $\mathrm{L}$. & 2 & 2 \\
\hline & Sorbus intermedia (Ehrh.) Pers. & 2 & 2 \\
\hline & Taxus baccata $\mathrm{L}$. & 3 & 3 \\
\hline \multirow[t]{8}{*}{3} & (estrato arbustivo) & 2 & 2 \\
\hline & Corylus avellana $\mathrm{L}$. & 4 & 3 \\
\hline & Rhamnus alpina L. & 1 & 1 \\
\hline & Ribes alpinum L. & 1 & 1 \\
\hline & Rosa sp. & 1 & 1 \\
\hline & Sorbus aucuparia L. & 1 & 2 \\
\hline & Taxus baccata L. & 1 & 1 \\
\hline & Viburnum lantana L. & 1 & 1 \\
\hline \multirow[t]{9}{*}{2} & (estrato subarbustivo) & 2 & 2 \\
\hline & Cytisus cantabricus (Willk.) Rchb. F. & 1 & 1 \\
\hline & Daphne laureola L. s.l. & 1 & 1 \\
\hline & Genista hispanica L. subsp. occidentalis Rouy & 1 & 1 \\
\hline & Iris latifolia (Mill.) Voss & 1 & 1 \\
\hline & Lilium martagon L. & 1 & 1 \\
\hline & Polystichum aculeatum (L.) Roth & 1 & 1 \\
\hline & Ribes alpinum L. & 1 & 1 \\
\hline & Thalictrum minus L. & 1 & 1 \\
\hline \multirow[t]{20}{*}{1} & (estrato herbáceo) & 2 & 2 \\
\hline & Arabis alpina $\mathrm{L}$. & 1 & 1 \\
\hline & Asplenium adiantum-nigrum L. & 1 & 1 \\
\hline & Brachypodium pinnatum subsp. rupestre (Host) Schübl. \& G. Martens & 1 & 1 \\
\hline & Carex brevicollis DC. & 1 & 1 \\
\hline & Echium vulgare $\mathrm{L}$. & 1 & 1 \\
\hline & Euphorbia flavicoma DC. & 1 & 1 \\
\hline & Galium mollugo L. & 1 & 1 \\
\hline & Geranium robertianum L. & 1 & 1 \\
\hline & Helianthemum nummularium (L.) Mill. & 1 & 1 \\
\hline & Helleborus viridis subsp. occidentalis (Reut.) & 1 & + \\
\hline & Hepatica nobilis Schreb. & 1 & 1 \\
\hline & Melica nutans L. & 1 & 1 \\
\hline & Mercurialis perennis $\mathrm{L}$. & 1 & 1 \\
\hline & Pentaglottis sempervirens (L.) Tausch ex L. H. Bailey & 1 & 1 \\
\hline & Polypodium vulgare L. & 1 & 1 \\
\hline & Primula veris subsp. columnae (Ten.) Maire \& Petitm. & 1 & 1 \\
\hline & Sedum anglicum Huds. & 1 & 1 \\
\hline & Sideritis hyssopifolia L. s.I. & 1 & 1 \\
\hline & Teucrium pyrenaicum $\mathrm{L}$. & 1 & 1 \\
\hline
\end{tabular}


Inventarios en roquedos de Vatsongo (1) y Valdesiniestro (2)

\begin{tabular}{|c|c|c|c|}
\hline & \\
\hline & & 1 & 2 \\
\hline 5 & (estrato arbóreo) & 0 & 0 \\
\hline 4 & (estrato arborescente) & 3 & 1 \\
\hline & Corylus avellana $\mathrm{L}$. & 3 & - \\
\hline & Sorbus aria (L.) Crantz & 1 & - \\
\hline & Taxus baccata $\mathrm{L}$. & 2 & 5 \\
\hline 3 & (estrato arbustivo) & 2 & 2 \\
\hline & Berberis vulgaris subsp. cantabrica Rivas Mart., T.E. Díaz, Fern.Prieto... & 1 & 1 \\
\hline & Corylus avellana $\mathrm{L}$. & 4 & - \\
\hline & Daphne laureola L. s.I. & - & 1 \\
\hline & Genista hispanica L. subsp. occidentalis Rouy & - & 2 \\
\hline & Heracleum sphondylium L. s.I. & - & 1 \\
\hline & Laserpitium latifolium L. & - & 1 \\
\hline & Rhamnus alpina L. & 1 & 4 \\
\hline & Ribes alpinum L. & - & 1 \\
\hline & Rosa sp. & 1 & - \\
\hline & Taxus baccata L. & 1 & 3 \\
\hline & Viburnum lantana L. & 1 & - \\
\hline 2 & (estrato subarbustivo) & 2 & 3 \\
\hline & Berberis vulgaris subsp. cantabrica Rivas Mart., T.E. Díaz, Fern.Prieto... & 1 & 2 \\
\hline & Corylus avellana $\mathrm{L}$. & 1 & - \\
\hline & Crepis lampsanoides Froel. & - & 1 \\
\hline & Cytisus cantabricus (Willk.) Rchb. F. & 1 & 1 \\
\hline & Daphne laureola L. s.I. & 1 & 2 \\
\hline & Erica vagans $\mathrm{L}$. & - & 1 \\
\hline & Euphorbia hyberna L. & - & 1 \\
\hline & Genista hispanica L. subsp. occidentalis Rouy & 1 & 1 \\
\hline & Iris latifolia (Mill.) Voss & 1 & - \\
\hline & Juniperus alpina (Suter) S.F. Gray & - & 1 \\
\hline & Lilium martagon $\mathrm{L}$. & 1 & - \\
\hline & Polystichum aculeatum (L.) Roth & 1 & 1 \\
\hline & Ribes alpinum L. & 1 & 2 \\
\hline & Thalictrum minus L. & 1 & 1 \\
\hline 7 & (estrato herbáceo) & 3 & 4 \\
\hline & Arenaria grandiflora $\mathrm{L}$ & - & 1 \\
\hline & Brachypodium pinnatum subsp. rupestre (Host) Schübl. \& G. Martens & 1 & 1 \\
\hline & Carex brevicollis DC. & - & 1 \\
\hline & Crepis albida Vill. subsp. asturica (Lacaita \& Pau) Babc. & - & 1 \\
\hline & Echium vulgare $\mathrm{L}$. & 1 & - \\
\hline & Euphorbia flavicoma DC. & 1 & - \\
\hline & Galium mollugo L. & 1 & - \\
\hline & Geranium robertianum L. & - & 1 \\
\hline & Glandora prostrata (Loisel.) D.C.Thomas & - & 1 \\
\hline & Helianthemum nummularium (L.) Mill. & 1 & 1 \\
\hline & Helleborus viridis subsp. occidentalis (Reut.) & 1 & - \\
\hline & Hepatica nobilis Schreb. & 1 & 1 \\
\hline & Mercurialis perennis $\mathrm{L}$. & 1 & 1 \\
\hline & Pentaglottis sempervirens (L.) Tausch ex L. H. Bailey & 1 & - \\
\hline & Polypodium vulgare L. & 1 & - \\
\hline & Primula veris subsp. columnae (Ten.) Maire \& Petitm. & 1 & - \\
\hline & Saxifraga paniculata Mill. & - & 1 \\
\hline & Saxifraga trifurcata Schrad. & - & 1 \\
\hline & Scabiosa columbaria L. & - & 1 \\
\hline & Sideritis hyssopifolia L. s.I. & 1 & - \\
\hline & Teucrium pyrenaicum $\mathrm{L}$. & 1 & 1 \\
\hline
\end{tabular}

